\title{
Risk based Test Case Prioritization using UML State Machine Diagram
}

\author{
Gufran Ahmad Ansari, \\ PhD \\ Assistant Professor, \\ Department of Information \\ Technology, College of \\ Computer, Qassim University, \\ Al-Qassim, Kingdom of Saudi \\ Arabia(KSA)
}

\author{
Wasiur Rhmann \\ Department of Computer \\ Science, Babasaheb Bhimrao \\ Ambedkar University (A \\ Central University), Lucknow, \\ India
}

\author{
Vipin Saxena \\ Department of Computer \\ Science, Babasaheb Bhimrao \\ Ambedkar University (A \\ Central University), Lucknow, \\ India
}

\begin{abstract}
Time and budget constraints are two main factors which affect the quality of software products. Testing is the last activity that performed in the software development life cycle which suffers more. Test cases are designed to find the bugs in the software. Prioritization of test cases is used to execute test cases earlier which are of high probability of finding bugs. Testing with all possible test cases is not possible even for trivial software. Testing of safety critical systems require more effort to identify the critical components. In the present work, authors proposed, a novel technique of test cases prioritization from UML state diagram by taking account risk. State machine diagram is transformed into WEFSM (Weighted Extended Finite State Machine) and a case study of ATM system is used to evaluate the proposed approach. A new metric based on risk called APRC (Average Percentage of Risk Covered) is proposed and used to demonstrate the effectiveness of proposed approach.
\end{abstract}

\section{Keywords}

Software Testing, Test Cases, State Diagram, Risk Exposure, UML

\section{INTRODUCTION}

Now a day's safety critical systems are heavily used in different domain of life like health, real-time embedded and financial systems. Some functions of these critical systems are more important and error prone than other and these critical functions are needed to be tested properly to ensure the correctness of the functionality. Test cases should be designed in such a way that can expose the defects in these critical functions of the software. Software testing is a validation activity of the software product. Test cases are designed with the intention of fault detection. Test case is defined [1] as triplet [I, S, O] where I is input to the system, S is state of the system at which input is given and $\mathrm{O}$ is expected output. Test cases can be designed from either code of the software or design and specification documents. As software testing is usually last activity performed in the software development process and it is often performed in the pressure. Risk management process which takes earlier the preventive measures cost negligible compared to huge cost that may incurred when use of proper risk management techniques is neglected [2]. Risk based testing considers both time and risk related to the software component. It focuses on the activities of the functionality of the software which can trigger hazard condition for a software system [3]. Risk based testing is emphasized on allocation of large time in testing of critical components of the software [4]. Unified modeling language is process-independent standard language for modeling objectoriented systems. It has become standard for designing high quality software and it is also used for security analysis along with the designing the software systems [5]. Different UML diagrams are used to model different views of the system. State machine represents the sequences of states of an object in which it goes through during its lifetime in response to events together with its responses to those events [6]. State diagram consists of states and transitions between different states. States are represented by rounded rectangle and transition between different states is represented by arrow connecting them. Objects which behave differently based on its state can be suitably model with the state diagram [7]. Prioritization of test cases schedules the execution of test cases in such a way that test cases with high probability of fault detection are executed first. Test cases prioritization can detect fault earlier. UML models are used by researchers and industry person in different domains for different purpose. In [8-9] UML models are used for effort estimation and in [1011] risk estimation is performed. In this article, we used UML state diagram to model the software system and attached risk information associated with different states of the system. The risk information is calculated based on risk exposure. Each risk is associated with different state. After that UML state diagram is converted into weighted extended finite state machine (WEFSM) and prioritized test cases are generated using risk exposure information.

\section{RELATED WORK}

Hettiaracchichi et al. [12] have used system requirements and risk associated with the requirements for identification of important test cases. Fuzzy expert system is used to make the risk estimation less subjective. Risk modification, complexity, security and size of software requirements are used as risk indicators. Proposed approach can identify the faults in the system earlier. Stallbaum et al. [13] presented a risk based testing called RiteDAP. Authors used UML activity diagram and prioritized the generated test cases based on risk. Test cases are prioritized based on fault probability and damage due to that fault. Proposed technique is applied on a real example. Gebizi et al. [14] have used an iterative method for model based testing using risk information. Authors used Morkov chain model for system under test. Initially equal probabilities are assigned to each transition. Memory leaks are used to assign updated transition probabilities based on failure. Smart TV system is used to validate the proposed approach. Shirole et al. [15] have demonstrated UML state 
diagram as specification of the system. State diagram is used to represent the dynamic aspect of the system. Authors converted the diagram into extended flow graph and from extended flow graph feasible paths are generated using genetic algorithm. Mohanty et al. [16] presented a regression test case prioritization technique for component based software using models. Authors used state chart diagram for representation of components and changes of components. State chart diagram is converted into component interaction graph (CIG). CIG and old test suite is taken as input and used information about total state changes direct and indirect access of database from test cases is used for test cases prioritization. Proposed approach minimized the cost of system testing. Samuel et al. [17] have presented an approach of automatic test cases generation from UML state diagram. Authors utilized the control and data flow information of the state diagram. State diagram is traversed and conditional nodes are selected. Functional minimization technique is used for generation of test data from the selected conditional nodes. Cluster level behavior can be tested with the generated test cases.

\section{BACKGROUND}

\subsection{Risk Measurement}

Risk is an expectation of loss that may or may not occur for any project. Software risk is due to the possibility of suffering loss in software projects. Loss can result the poor quality product, high cost and delay in completion of projects. In risk assessment different situations of systems and risk associated with those situations is determined. For quantitative calculation of risk, probability of a risk occurrence $(\mathrm{P})$ and possible loss (L) due to that risk are use.

Mathematically risk exposure is calculated by the following formula:

$\mathrm{RE}=\mathrm{P} * \mathrm{~L}$

(1)

Where RE is risk exposure, $\mathrm{P}$ is probability of risk/ hazard occurrence and $\mathrm{L}$ is loss due to hazard.

Some risks are tolerable as damage caused by that risk is less compared to applying effective measure to tackle that risk. In similar way some function or component of the software may be more prone to risk. Risk with high probability of occurrence and damage cost are treated in different way in comparison to lower probability of occurrence and damage cost.

Risk assessment categorized the following processes:

Identify the risks, Analyze the risk, Evaluate and prioritize the risk and Tackle the risk.

a. Identify the risks In this phase, external and internal events that can pose threat to projects are identified.

b. Analyze the risks Analyze various factors contributing to the risks.

c. Evaluate and prioritize the risks Prioritize and characterize risk in different categories.

d. Tackle the risks Identify different options to tackle the risk and implement the best one with the available resources.

\subsection{UML Elements used for Risk Information Representation}

It is not possible from limited elements of UML to facilitate the sufficiently express the entire model across different domain. UML provides mechanism to extend the vocabulary of UML for creation of new model elements. These new elements are derived from exiting elements of UML and are very useful for particular domain. There are three types of extensible mechanism in UML namely: Stereotype, Tags and Constraints. In the present work Stereotype and Tag values are used for representation of risk information.

\subsubsection{Stereotypes}

Unified Modeling Language provides the concept of stereotypes which can be used to modify the meaning of an element in UML and assign it to new role. It helps to create new types of building blocks from existing. Different stereotypes are used to represent different category of risk.

\subsubsection{Tag Values}

Properties of a UML building block can be extended with the tagged value. New information in an element specification can be created by tagged value. In the presented work probability of risk occurrence and damage cost is represented in tag values for each category of risk.

\subsection{Weighted Extended Finite State Machine (WEFSM)}

A WEFSM (Weighted Extended Finite State Machine) is in which nodes are used to represent states of the system and edges represent the transition between states. A WEFSM consists of the following elements:

States- States are conditions of the system

Extended States- States with associated variable or memory is extended state.

Guards-Guards are Boolean conditions attached with transitions which enable the change of states.

Events- Events are incidents which change the state of the system.

Actions- Actions are performed when an event occurs.

Transitions-Transitions from one state to other state occur due to events and may have guards.

Weights- A weight (risk exposure) is associated with each transition edge.

\section{PROPOSED METHODOLOGY}

The presented approach is summarized in the following steps:

1. Draw a UML state diagram of the software system;

2. Identify the states of UML state diagram from which risk may be associated and identify the risk;

3. Categorize the risk and compute the risk exposure for each risk;

4. Redraw the UML state diagram and attach the risk associated with each state using tags and stereotypes of the UML diagram.

5. Transform the UML state diagram obtained in step4 to WEFSM (Weighted Extended Finite State Machine). 
Risk information is used to assign weights to the WEFSM.

6. From the weighted extended finite state machine draw weighted state transition table. Weighted State Transition Table (WSTT) contains the risk exposure information for each state.

7. Use the weighted state transition table (WSTT) for generation of test cases.
8. Sort the test cases in descending order of risk exposure.

\section{A CASE STUDY: ATM}

In the present work we skipped the normal UML state diagram without the risk information and UML state diagram with risk information attached with different state is presented in Figure 1. Risk information attached with different states is represented with Stereotypes and Tags. Customer can enquiry balance and withdraw balance.

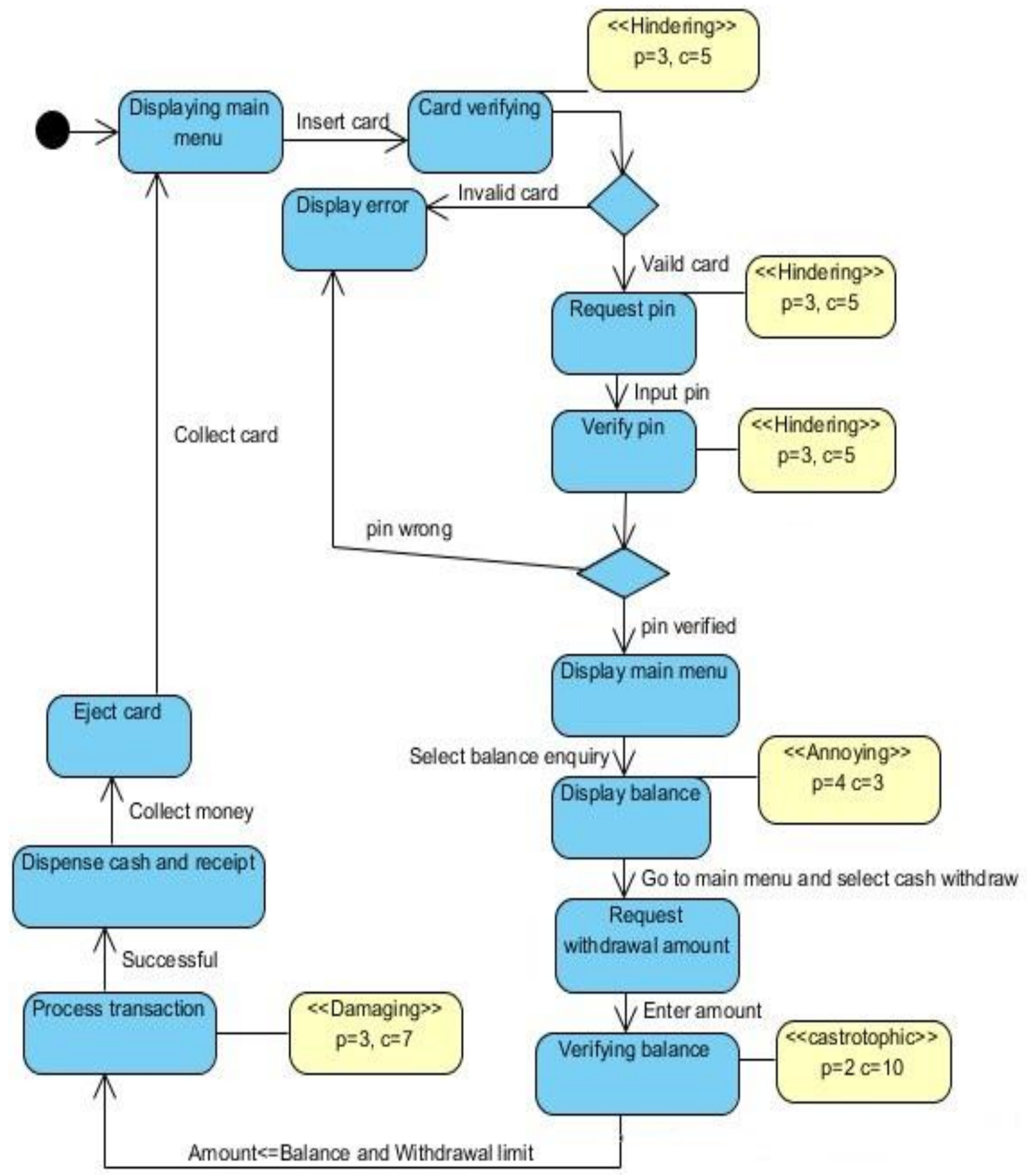

Figure 1. UML State Machine Diagram of ATM with risk information 
In the Table 1. risk is categorized based on Schaefer [18]. Risk types are written in descending order of severity [19]. If system is not performing balance enquires then it will be least damaging and risk will be attached with the state balance enquiry. If someone other than customer withdraws money for customer account above the withdrawal limit then it will be of highest severity and customer will be highly affected by it and such types of risk will be associated with the verifying balance state. Similarly, Normal withdrawal and Invalid ATM and Pin entry failure are categorized in Damaging and hindering type of risk.

Table 1. Representation of risk and risk type associated with the state

\begin{tabular}{|l|l|l|}
\hline \multicolumn{1}{|c|}{ Risk Type } & Risk & State \\
\hline Catastrophic & Invalid withdrawal & Verifying balance \\
\hline Damaging & $\begin{array}{l}\text { Normal } \\
\text { withdrawal }\end{array}$ & $\begin{array}{l}\text { Process } \\
\text { transaction }\end{array}$ \\
\hline Hindering & $\begin{array}{l}\text { Invalid ATM, Pin } \\
\text { entry failure }\end{array}$ & $\begin{array}{l}\text { Request pin, } \\
\text { verify card }\end{array}$ \\
\hline Annoying & Balance enquires & Balance enquiry \\
\hline
\end{tabular}

High damaging cost is assigned to catastrophic risk and low cost is assigned for annoying risk while probability of occurrence of catastrophic risk is low and high for annoying risk types. In Table 2. different states with associated risks and their risk exposure is calculated, probability of risk occurrence is given as 2, 3, 3, 4 and cost of damage is given as $10,7,5,3$ for catastrophic, damaging, hindering and annoying.

Table 2. Representation of state and associated risk with state with risk exposure

\begin{tabular}{|l|l|l|}
\hline State & Risk types & $\mathbf{R E}=\mathbf{P}^{*} \mathbf{C}$ \\
\hline Verifying balance & Catastrophic & $2 * 10=20$ \\
\hline Process transaction & Damaging & $3 * 7=21$ \\
\hline $\begin{array}{l}\text { Request pin, verify } \\
\text { card }\end{array}$ & Hindering & $3 * 5=15$ \\
\hline Balance enquiry & Annoying & $4 * 3=12$ \\
\hline
\end{tabular}

Figure 2 represents the WEFSM (Weighted Extended Finite State Machine). It is a directed graph drawn from UML State Diagram of Figure 1. For each state of the state diagram there is a node in WEFSM and edge represents the events that trigger the transfer from one state to other state. Edges of the directed graph are assigned weight based on risk exposure value of the states. There are 12 states in the state diagram in Figure. 1 these states are represented as nodes of the directed graph. States 2 and 5 contains two outgoing edges so in WEFSM there are two nodes with out-degree of 2 .

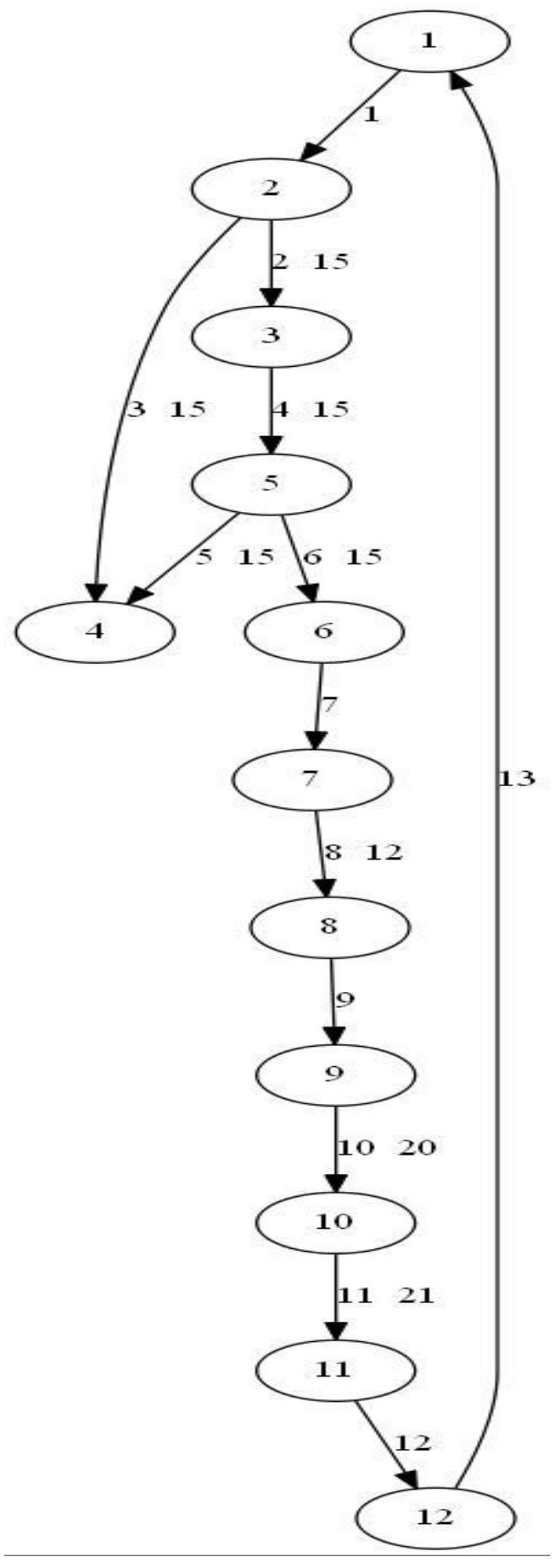

Figure 2. Weighted Extended Finite State Machine (WEFSM)

Weighted state transition table is drawn from WEFSM. States are written in left most column and events are written in upper most row of the table and for each state risk exposure is written in corresponding row. Risk exposure values are taken from UML state diagram. 
Table 3. Weighted State Transition Table (WSTT)

\begin{tabular}{|c|c|c|c|c|c|c|c|c|c|c|c|c|c|c|c|}
\hline Events & 1 & 2 & 3 & 4 & 5 & 6 & 7 & 8 & 9 & 10 & 11 & & 12 & 13 & RE \\
\hline States & \multicolumn{14}{|c|}{ Next States } & \\
\hline 1 & 2 & & & & & & & & & & & & & & 0 \\
\hline 2 & & 3 & 4 & & & & & & & & & & & & 15 \\
\hline 3 & & & & 5 & & & & & & & & & & & 15 \\
\hline 4 & & & & & & & & & & & & & & & 0 \\
\hline 5 & & & & & 4 & & 6 & & & & & & & & 15 \\
\hline 6 & & & & & & & & 7 & & & & & & & 0 \\
\hline 7 & & & & & & & & & 8 & & & & & & 12 \\
\hline 8 & & & & & & & & & & 9 & & & & & 0 \\
\hline 9 & & & & & & & & & & & 10 & & & & 20 \\
\hline 10 & & & & & & & & & & & & 11 & & & 21 \\
\hline 11 & & & & & & & & & & & & & 12 & & 0 \\
\hline 12 & & & & & & & & & & & & & & 1 & 0 \\
\hline
\end{tabular}

Test cases are designed from WSTT and presented in Table 4. Each test case consists of test case id, state, Event and next state and output of the next state and weight of each test case.

Table. 4 Test Cases

\begin{tabular}{|c|c|c|c|c|c|}
\hline $\begin{array}{l}\text { Test } \\
\text { case }\end{array}$ & State & EventlState & Output & $\begin{array}{l}\text { Next } \\
\text { State }\end{array}$ & Weight \\
\hline TC1 & 1 & 1 & & 2 & 0 \\
\hline TC2 & 2 & 2 & & 3 & 15 \\
\hline TC3 & 2 & 3 & $\begin{array}{c}\text { Please } \\
\text { enter } \\
\text { valid } \\
\text { card }\end{array}$ & 4 & 15 \\
\hline TC4 & 3 & 4 & $\begin{array}{c}\text { Please } \\
\text { enter } \\
\text { valid pin }\end{array}$ & 5 & 15 \\
\hline TC5 & 5 & 5 & & 6 & 15 \\
\hline TC6 & 5 & 6 & & 4 & 15 \\
\hline TC7 & 6 & 7 & & 7 & 0 \\
\hline TC8 & 7 & 8 & & 8 & 0 \\
\hline TC9 & 8 & 9 & & 9 & 12 \\
\hline TC10 & 9 & 10 & & 10 & 20 \\
\hline TC11 & 10 & 11 & $\begin{array}{c}\text { Collect } \\
\text { cash and } \\
\text { receipt }\end{array}$ & 11 & 21 \\
\hline TC12 & 11 & 12 & & 12 & 0 \\
\hline TC13 & 12 & 13 & $\begin{array}{c}\text { Collect } \\
\text { card }\end{array}$ & 1 & 0 \\
\hline
\end{tabular}

Prioritized order of test cases will be $\mathrm{T}_{11}, \mathrm{~T}_{10}, \mathrm{~T}_{2}, \mathrm{~T}_{3}, \mathrm{~T}_{4}, \mathrm{~T}_{5}$, $\mathrm{T}_{6}, \mathrm{~T}_{9}, \mathrm{~T}_{1}, \mathrm{~T} 7, \mathrm{~T}_{8}, \mathrm{~T}_{12}$, and $\mathrm{T}_{13}$.
A new metric called APRC (Average Percentage of Risk Covered) is proposed to demonstrate the effectiveness of the proposed approach and given below;

$$
A P R C=1-\frac{T R 1+T R 2+\cdots T R m}{n m}+\frac{1}{2 n}
$$

Where m-> Total number of risk

n-> Number of test cases, $T R_{i}$ ( The position of first test that covers the risk i) where $\mathrm{i}=1,2, . ., \mathrm{m}$

\section{APFC (Average Percentage of Risk Covered For}

\section{Prioritized Test Suite)}

$\mathrm{n}=13, \mathrm{~m}=8$

$$
\mathbf{A P F C}=1-\frac{1+2+3+4+5+6+7+8}{8 * 13}+\frac{1}{26}=0.6153
$$

APFC (Average Percentage of Risk Covered For NonPrioritized Test Suite)

$$
\mathrm{APFC}=1-\frac{11+10+2+3+4+5+6+9}{8 * 13}+\frac{1}{26}=0.480769
$$

Hence risk covered by prioritized test suite is larger than nonprioritized test suite which will increase the confidence in testing. 


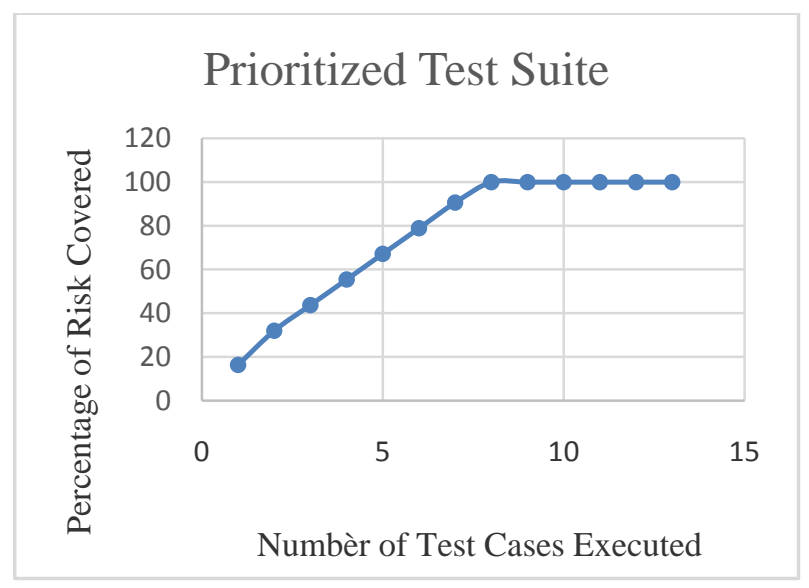

Figure 3. Number of test cases executed vs. risk covered for prioritized test suite

Figure 3. and Figure 4. represent the number of test cases executed and percentage of risk covered by executed test cases in the graphical form. In case of prioritized test suite total risk is covered by executing only 8 test cases while in case of non-prioritized test suite, total risk is covered with the execution of $11^{\text {th }}$ test case. Figure 3 shows that risk is covered earlier in comparison to non-prioritized test suite which is presented in Figure 4.

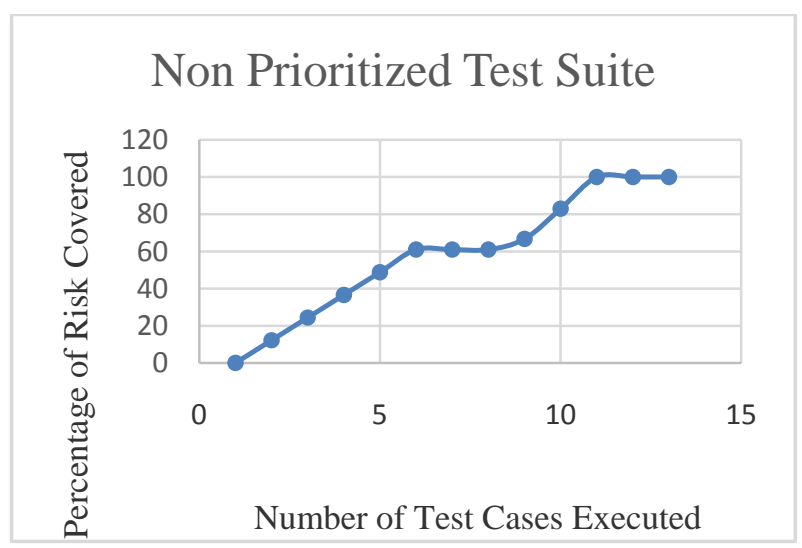

Figure 4. Number of test cases executed vs. risk covered for non-prioritized test suite

\section{CONCLUSIONS}

Risk analysis is inevitable for safety critical applications. If risk analysis can be done earlier in the development process then suitable risk mitigation approach can be applied to reduce the damage. UML is used by software engineer to design high quality software and risky components can easily identify by using UML. In the present work UML state machine diagram is used represent various states of the system. Various researchers used UML state diagram for test cases generation but very few work has been reported by taking into account the risk associated with the project. Risk is identified for each state and these risks are attached with those states and risk exposure values are computed for each those state. Test cases with higher risk are given higher priority. Results indicate that risk covered by test cases generated by presented approach is higher than the non-prioritized test suite. Identification of risky components earlier will boost the confidence of tester and also improve the reliability of the software. Proposed approach will be suitable for testing safety critical applications and application in which some functions are more error and risk prone. This technique may also helpful for health related software where hazard may injure patient and sometime life may be put in danger. In future cluster level test cases from UML interaction diagram with consideration of risk may be designed and a fully automatic tool may also be design.

\section{REFERENCES}

[1] Mall, R. 2009. Fundamental of Software Engineering, $3^{\text {rd }}$ Edition, Prentice Hall.

[2] McManus, J. 2004. Risk Management in Software Development Projects, First Edition, Elsevier.

[3] Amland, S. 2000. Risk Based Testing: Risk Analysis Fundamentals and Metrics for Software Testing including a Financial Application Case Study, Journal of Systems and Software, Vol. 53, No. 3, pp. 287-295.

[4] Wendland, M. F., Kranz, M. and Schieferdecker, I. 2012. A Systematic Approach to Risk-Based Testing Using Risk-annotated Requirements Models, International Conference on Software Engineering Advances, pp. 636642 .

[5] Bar, T., Strohmeier, A., Moreira, A. and Mellor, S. J. 2004. The Unified Modeling Language: Modeling languages and applications, International Conference on Lisbon, Portugal, Springer.

[6] Booch, G., Raumbagh, J. and Jaobson, I. 1998. The Unified Modeling Language User Guide, Addison Wesley.

[7] Hamilton, K. and Miles, R. 2006. Learning UML 2.0, O Reilly.

[8] Zivkovic, A., Rozman, I. and Hericko, M. 2005. Automated Software Size Estimation Based on Function Points using UML Models, Information and Software Technology, Vol. 47, pp. 881-890.

[9] Hakim, A. S. H., Abran, A. and Abdallah, H. B. 2015. A Measurement Method for Sizing the Structure of UML Sequence Diagrams, Information and Software Technology, Vol. 59, pp. 222-232.

[10] Popstoiannova, K. G., Hassan, A., Guedem, A., Abdelmoez, W., Nassar, D. E. M., Ammar, H. and Milli, A. 2003. Architecture Level Risk Analysis using UML, IEEE Transaction on Software Engineering, Vol. 29, No. 10, pp. 2003.

[11] Wang, T., Hassan, A., Guedem, A., Popstojanaova, K. G. and Ammar, H. 2003. Architectural Level Risk Assessment Tool Based on UML Specification, International Conference on Software Engineering, IEEE, pp. 808-809.

[12] Hettiarachichi, C., Do, H. and Choi, B., 2016. Risk Based Test Case Prioritization Using a Fuzzy Expert System, Information and Software Technology, Vol. 69, pp. 1-15.

[13] Stallbaum, H., Metzqer, A. and Pohl, K. 2008. An Automated Technique for Risk-Based Test Case Generation and Prioritization, Proceedings of $3^{\text {rd }}$ International Workshop on Automation of Software Test, pp. 67-70. 
[14] Gebizi, C. S., Metin, D. and Sozer, H. 2015. Combining Model Based Testing and Risk Based Testing for Effective Test Case Generation, Eighth International Conference on Softwate Testing, Verification and Validation Workshop, pp. 1-4.

[15] Shirole, M., Suthar, A. and Kumar, R. 2011. Generation of Improved Test Cases from UML State Diagram using Genetic Algorithm, Proceeding of Indian Software Engineering Conference, ACM, pp. 125-134.

[16] Mohanty, S., Acharya, A. A. and Mohpatra, D. P. 2011. A Model Based Prioritization Technique for Component Based Software Retesting using UML State Chart
Diagram, International Conference on Electronics Computer Technology, pp. 364-368.

[17] Samuel, P., Mall, R. and Bothra, A. K. 2008. Automatic Test Cases Generation From Unified Modeling Language (UML) State Diagrams, IET Software, Vol. 2, No. 2, pp. 79-93.

[18] Schaefer, H., Risk Based Testing, Strategies for Prioritizing Tests against Deadlines, Software Test Consulting, http://home.c2i.net/schaefer/testing.html, 2005.

[19] Jorgensen, P. C. 2014. Software Testing a Craftsman's Approach, $4^{\text {th }}$ edition, CRC Press. 